\title{
Induction of Dog Sperm Capacitation by Glycosaminoglycans and Glycosaminoglycan Amounts of Oviductal and Uterine Fluids in Bitches
}

\author{
Eiichi KAWAKAMI, Toshiro ARAI ${ }^{1)}$, Iwao OISHI ${ }^{1}$, Tatsuya HORI and Toshihiko TSUTSUI \\ Departments of Reproduction and ${ }^{11}$ Biochemistry, Nippon Veterinary and Animal Science University, 1-7-1 Kyonan-cho, Musashino-shi, \\ Tokyo 180-8602, Japan
}

(Received 28 May 1999/Accepted 14 September 1999)

\begin{abstract}
Ejaculated sperm collected from 12 beagle dogs were incubated in canine capacitation medium (CCM), supplemented with 5 $\mu \mathrm{g} / \mathrm{m} l$ chondroitin sulfate A (CS), $5 \mu \mathrm{g} / \mathrm{m} l$ hyaluronic acid (HA), or $5 \mu \mathrm{g} / \mathrm{m} l$ heparin (HP) for $7 \mathrm{hr}$ at $38^{\circ} \mathrm{C}$ in a $5 \% \mathrm{CO}_{2}$ in air atmosphere to investigate the effects of glycosaminoglycans (GAGs) on dog sperm capacitation. The percentages of motile sperm, hyperactivated sperm (\%HY), and acrosome-reacted sperm (\%AR) in all media were examined after $4 \mathrm{hr}$ and $7 \mathrm{hr}$ of incubation. The oviducts and uteri of 9 anestrous and 18 estrous beagle bitches were removed under halothane inhalation anesthesia to measure the total GAG amounts in oviductal and uterine fluids. The lumens of the ampulla of the oviducts, isthmus of the oviducts, and the uterine horns were each flushed with $1 \mathrm{~m} l$ HEPES-EDTA fluid. Total GAG amounts in the flush fluids obtained were measured with a spectrophotometer. Sperm motility (51-59\%), \%HY (79-86\%), and \%AR (31-36\%) in CCM supplemented with CS, HA, or HP were significantly higher after $7 \mathrm{hr}$ of incubation than when incubated in CCM without GAGs $(\mathrm{P}<0.01$ or 0.05$)$. The mean total GAG amounts in the fluids from the ampulla and isthmus of the oviducts and the uterine horns in the estrous bitches were higher than in the anestrous bitches. These results indicate that GAGs in the oviductal and uterine fluids in estrous bitches are associated with in vivo sperm capacitation. —KEY wORDS: canine, capacitation, glycosaminoglycan, sperm.
\end{abstract}

Sperm capacitation has been found to be effectively induced in the oviducts of many species [4, 6, 13, 35], and it has been reported that a large volume of glycosaminoglycans (GAGs) in oviductal fluid may play a major role in bovine sperm capacitation in estrous cows [2, 8, 18, 29]. GAGs are mucopolysaccharides and include chondroitin sulfate A (CS), hyaluronic acid (HA), and heparin (HP) [19, 22]. Addition of CS [19, 25], HA [33], or HP $[9,21,28]$ to bovine sperm medium has been found to stimulate motility and capacitation of sperm, and HP and oviductal fluid collected from cows have been reported to cause functional changes in the bovine sperm cell membrane [21] and activation of cyclic AMP [30]. In addition, HA has been found to induce human sperm capacitation by improving of $\mathrm{Ca}$ absorption into the sperm [34]. HAbinding protein [31] and HP-binding protein [23] have been found to be present on the bovine sperm cell membrane, and mucoproteins in cow oviductal fluid have been discovered to induce changes in the bovine sperm cell membrane in relation to capacitation after binding to the sperm surface [24].

The authors have observed that addition of oviductal flush fluid collected from estrous bitches to canine sperm medium is very effective in inducing sperm capacitation [15]. However, there have been no reports on the effects of GAGs on dog sperm capacitation or the amounts of GAG in oviductal and uterine fluids of bitches. In the present study, the effects of addition of CS, HA, or HP to canine sperm medium on sperm capacitation were examined, and total GAG amounts in flush fluids from the oviducts and uterine horns of anestrous and estrous bitches were measured to investigate the relationship between in vivo capacitation of dog sperm and the concentrations of GAG.

\section{MATERIALS AND METHODS}

Animals: Twelve male beagle dogs, aged 2-6 years with normal semen quality, cared for in our university, were used to collect the semen for this experiment. The dogs were housed in pens with ample runs under natural lighting. Commercial dry dog food was provided twice a day and access was given to water ad libitum. Nine anestrous and eighteen estrous beagle bitches, aged $4-8$ years, cared for in our university and at a pet food company, were subjected to ovariohysterectomy under halothane inhalation anesthesia.

Semen collection and evaluation: The sperm-rich second fraction of ejaculated semen [10] was collected by digital manipulation and immediately transported to our laboratory. The concentration of sperm in the semen was determined by hematocytometer counts. Sperm motility, i.e., the percentage of actively motile sperm, was evaluated by using a sperm motility examination plate and a warm-plate. Semen samples with excellent sperm motility (>90\%) were used.

Sperm incubation: The spermatozoa in the semen were washed twice by centrifugation at $400 \mathrm{~g}$ for $5 \mathrm{~min}$ in $5 \mathrm{ml}$ of canine capacitation medium (CCM) [20] warmed to $38^{\circ} \mathrm{C}$. The final sperm pellet was diluted in CCM supplemented with $5 \mu \mathrm{g} / \mathrm{m} l \mathrm{CS}, 5 \mu \mathrm{g} / \mathrm{m} l \mathrm{HA}$, or $5 \mu \mathrm{g} / \mathrm{m} l$ HP to a concentration of $5 \times 10^{6} \mathrm{sperm} / \mathrm{m} l$. It has been reported that $10 \mu \mathrm{g} / \mathrm{m} l \mathrm{HA}$ for hamster sperm [22] and $10 \mu \mathrm{g} / \mathrm{m} l$ HP for bovine sperm [25] incubation were used. Therefore, the 
effects of $5 \mu \mathrm{g} / \mathrm{ml}$ GAGs on in vitro capacitation of dog sperm were examined. CCM without CS, HA, or HP was used as the control. Sperm incubation was performed in $120 \times 15 \mathrm{~mm}$ glass test tubes, and the tubes were loosely capped and incubated for $7 \mathrm{hr}$ at $38^{\circ} \mathrm{C}$ in an atmosphere of $5 \% \mathrm{CO}_{2}$ in air.

Evaluations of hyperactivated and acrosome-reacted sperm: Sperm motility and the percentages of hyperactivated sperm $(\% \mathrm{HY})$ and acrosome-reacted sperm $(\% \mathrm{AR})$ in the four types of CCM described above were determined $4 \mathrm{hr}$ and $7 \mathrm{hr}$ after the start of incubation. The \% $\mathrm{HY}$ and \%AR were estimated by counting the number of sperm with starspin-like movement in a fixed position among all motile sperm [16] and by the triple-stain technique [36], respectively.

Flushing of oviducts and uteri: The right sides of the oviducts and uterine horns removed from the anestrous and estrous bitches were used. The ampulla and isthmus of the oviducts were divided. A 20-gauge retaining needle connected to a $1-\mathrm{m} l$ injection syringe was inserted into the lumen of the ampulla and isthmus of oviducts and the uterine horns, and then the lumen of each portion was flushed with $1 \mathrm{~m} l$ of HEPES-EDTA solution (25 mM HEPES and $1 \mathrm{mM}$ EDTA, pH7.3). The flush fluids were collected in test tubes.

Measurement of GAG amounts: Total GAG concentrations in the flush fluids of the ampulla and isthmus of the oviducts, and the uterine horns were measured according to the method described by Whiteman [38]. Briefly, $0.05 \%$ Alcian Blue reagent with $50 \mathrm{mM}$ sodium acetate was centrifuged at $200 \mathrm{~g}$ for $15 \mathrm{~min}$, and the precipitate was removed by aspiration. The Alcian Blue solution $(1 \mathrm{ml})$ was added into the sample tubes and the standard tubes each, and the tubes were allowed to stand at room temperature for $2 \mathrm{hr}$. The Alcian Blue-GAG solution was centrifuged at $200 \mathrm{~g}$ for $15 \mathrm{~min}$, the supernatant solution was removed by decantation, and the Alcian Blue-GAG complex was washed with $2 \mathrm{~m} l$ of absolute ethanol by centrifugation. Manoxol IB reagent $(1 \mathrm{~m} l)$ was added to all tubes after removal of the supernatant, and the total GAG amounts in the tubes was measured with a spectrophotometer at an absorbance $620 \mathrm{~nm}$.

Statistical analysis: The data are summarized as mean values \pm standard error(S.E.) or standard deviation (S.D.). Differences between means were analyzed statistically by Student's $t$-test.

\section{RESULTS}

Capacitation of sperm incubated with GAGs: Changes in the sperm motility, \%HY, and \% AR of dog sperm incubated in CCM containing CS, HA, or HP are shown in Figs.1, 2, and 3. The mean values for sperm motility in CCM with GAGs both $4 \mathrm{hr}$ and $7 \mathrm{hr}$ after the start of incubation were significantly higher than the sperm motility values in the control CCM $(\mathrm{P}<0.01$ or $\mathrm{P}<0.05)$. Similarly, both the \%HY and \%AR in CCM with GAGs at both $4 \mathrm{hr}$ and $7 \mathrm{hr}$ after the start of incubation were significantly higher than in the

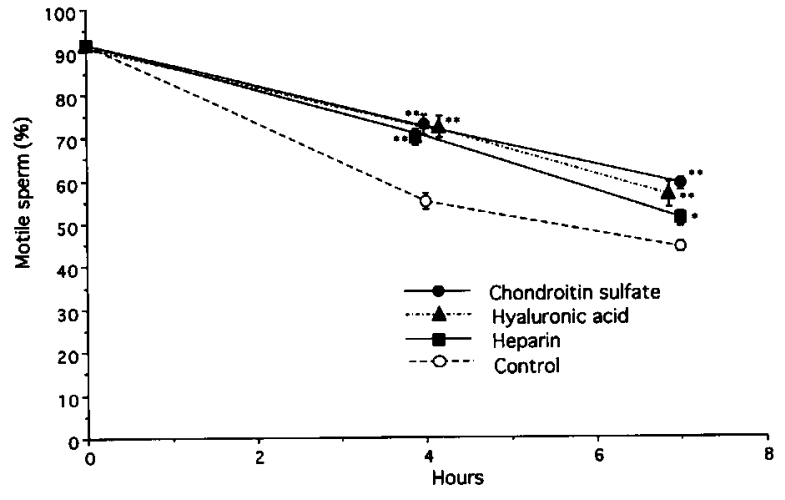

Fig. 1. Changes in the mean $( \pm$ S.E.) percentages of motile sperm of 12 beagle dogs in CCM supplemented with glycosaminoglycans.

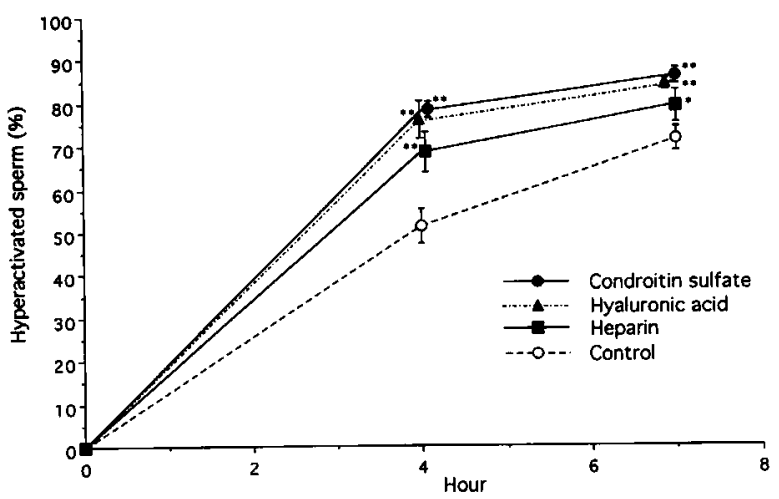

Fig. 2. Changes in the mean ( \pm S.E.) percentages of hyperactivated sperm of 12 beagle dogs in CCM supplemented with glycosaminoglycans.

control CCM $(\mathrm{P}<0.01$ or $\mathrm{P}<0.05)$. However, there were no differences in sperm motility, \%HY, or \% AR among spermatozoa treated with CS, HA and HP.

GAG amounts in the oviductal and uterine fluids: The mean ( \pm S.D.) total GAG amounts in the flush fluids of the ampulla of the oviducts, isthmus of the oviducts, and the uterine horns in anestrous and estrous bitches are shown in Table 1. All of the mean total GAG amounts in the estrous bitches were higher than in anestrous bitches, but there was no difference between the anestrous and estrous bitches in the GAG amounts in the flush from the ampulla and isthmus of oviducts and the uterine horns. Nor were there any differences between the amounts in the ampulla or isthmus of the oviducts, or the uterine horns in either the anestrous or estrous bitches.

\section{DISCUSSION}

It has been reported that the motility and capacitation of bovine sperm are effectively induced by addition of CS [19, 25], HA [33], or HP [9, 21, 28] to the sperm medium, and GAGs including CS, HA, and HP, have been found to cause 


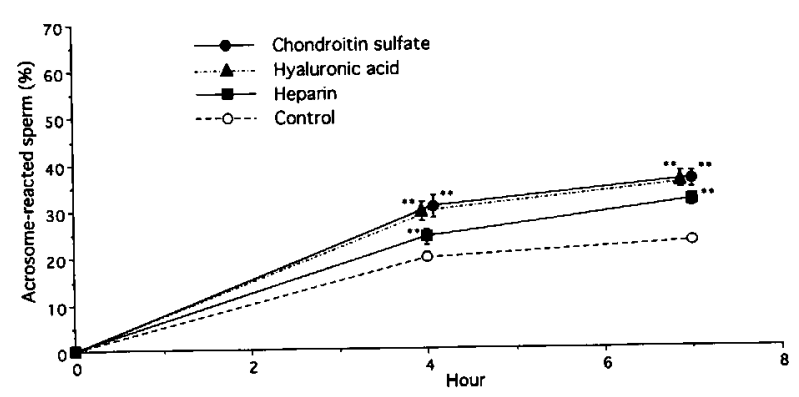

Fig. 3. Changes in the mean ( \pm S.E.) percentages of acrosomereacted sperm of 12 beagle dogs in CCM supplemented with glycosaminoglycans.

sperm capacitation as a result of inducing functional changes in sperm cell membrane [21], absorption of $\mathrm{Ca}$ into the sperm [24], and activation of cyclic AMP in the sperm [30]. The results of the present study demonstrated that maintenance of sperm motility and induction of in vitro sperm capacitation are caused by GAGs in the dog, the same results have been obtained in the bull, the hamster [22], goat [5], monkey [37], and humans [12]. The authors had previously found that hyaluronidase and $\mathrm{N}$ acetylhexosaminidase, the enzymes which hydrolyze GAGs, are present in dog sperm and that they are released during capacitation [14]. Thus, the GAGs in the fluid secreted by the genital organs of bitches may serve to accelerate the capacitation of dog sperm.

The secretory function of oviductal epithelial cells has been found to become active in the estrous period of different species $[1,18,27]$, and non-ciliated cells in the ampulla of the oviduct have been observed to secrete large amounts of glycoproteins in estrous ewes [27] and mice [26]. Furthermore, it has been reported that the oviductal fluid of estrous cows contains a large volume of GAGs [2, 8 , 17], and GAGs have been detected not only in the oviductal fluid of the cow [17] and ewe [18] but in their uterine fluid as well. The authors have verified that the addition of the oviductal flush fluid collected from estrous bitches to CCM maintains the sperm motility and induces capacitation of dog sperm [15]. In the present study, the total GAG amounts in the oviductal flush fluid of the estrous bitches was clearly higher than in the anestrous bitches, and presumably in vivo capacitation of dog sperm in estrous bitches is induced by the large volume of GAGs secreted from the oviductal epithelial cells. It has been reported that HA amounts in the lumen of the isthmus $(48-75 n \mathrm{~g} / \mathrm{ml})$ in the pig was much higher than in the ampulla $(10-23 \mathrm{ng} / \mathrm{ml})$ [32]. In the present study, however, there was no significant difference between total GAG amounts in the ampulla and isthmus of the oviducts in the bitches as well as total GAG amounts of the oviducts in the cows [2].

The GAGs were found not only in the oviductal fluid of bitches but in their uterine fluid. Synergism between the uterine and oviductal environment has been thought to effectively induce sperm capacitation within a short time in
Table 1. Total glycosaminoglycan amounts (ng/m $l$ Hepes-EDTA; $\mathrm{m} \pm$ S.D.) in the flush fluids collected from the ampulla and isthmus and the uterine horns in 9 anestrous and 18 estrous beagle bitches

\begin{tabular}{lccc}
\hline & \multicolumn{2}{c}{ Oviducts } & \multirow{2}{*}{ Uterine horns } \\
\cline { 2 - 3 } & Ampulla & Isthmus & \\
\hline Anestrous bitches & $152 \pm 28$ & $121 \pm 33$ & $141 \pm 21$ \\
Estrous bitches & $190 \pm 37$ & $166 \pm 54$ & $151 \pm 26$ \\
\hline
\end{tabular}

the rabbit [3] and pig [11], and dog sperm may also be capacitated by synergism between the uterus and oviduct in the bitch. Motile sperm have been observed in the uterine horn of a bitch 11 days after mating [7]. It was speculated that sperm motility in the uterus of the bitch is maintained over long periods by the effect of the GAGs in the uterine fluid. Based on the results of this study, it is concluded that GAGs in the oviductal and uterine fluids of estrous bitches are associated with in vivo capacitation of dog sperm.

\section{REFERENCES}

1. Abe, H., Sendai, Y., Satoh, T. and Hoshi, H. 1995. Bovine oviduct-specific glycoprotein: a potent factor for maintenance of viability and motility of bovine spermatozoa in vitro. Mol. Reprod. Dev. 42: 226-232.

2. Anderson, S.H. and Killian, G.J. 1994. Effect of macromolecules from oviductal conditioned medium on bovine sperm motion and capacitation. Biol. Reprod. 51: 795-799.

3. Bedford, J.M. 1969. Limitations of the uterus in the development of the fertilizing ability (capacitation) of spermatozoa. J. Reprod. Fertil. (Suppl.) 8: 19-26.

4. Cooper, G.W., Overstreet, J.W. and Katz, D.F. 1979. The motility of rabbit spermatozoa recovered from the female reproductive tract. Gamete Res. 2: 35-42.

5. Cox, J.F., Saravia, F., Briones, M. and Maria, A.S. 1995. Dose-dependent effect of heparin on fertilizing ability of goat spermatozoa. Theriogenology 44: 451-460.

6. Cummins, J.M. 1982. Hyperactivated motility patterns of ram spermatozoa recovered from the oviducts of mated ewes. Gamete Res. 6: 53-63.

7. Doak, R.L., Hall, A. and Dale, H.E. 1967. Longevity of spermatozoa in the reproductive tract of the bitch. J. Reprod. Fertil. 13: 51-58.

8. Grippo, A.A., Way, A.L. and Killian, G.J. 1995. Effect of bovine ampullary and isthmic oviductal fluid on motility, acrosome reaction and fertility of bull spermatozoa. J. Reprod. Fertil. 105: 57-64.

9. Handrow, R.R., Lenz, R.W. and Ax, R.L. 1982. Structual comparisons among glycosaminoglycans to promote an acrosome reaction in bovine spermatozoa. Biochem. Biophys. Res. Comm. 107: 1326-1332.

10. Harrop, A.E. 1955. Some observations on canine semen. Vet. Rec. 67: 494-498.

11. Hunter, R.H.F. and Hall, J.P. 1974. Capacitation of boar spermatozoa: synergism between uterine and tubal environments. J. Exp. Zool. 188: 203-214.

12. Huszar, G., Willetts, M. and Corrales, M. 1990. Hyaluronic acid (Sperm Select) improves retention of sperm motility and velocity in normospermic and oligospermic specimens. Fertil. 
Steril. 54: 1127-1134.

13. Katz, D.F. and Yanagimachi, R. 1980. Movement characteristics of hamster spermatozoa within the oviduct. Biol. Reprod. 22: 759-764

14. Kawakami, E., Arai, T. and Oishi, I. 1999. Changes in hyaluronidase, acrosin, and $\mathrm{N}$-acetylhexosaminidase activities of dog sperm after incubation. J. Vet. Med. Sci. 61: 183-184.

15. Kawakami, E., Hori, T. and Tsutsui, T. 1998. Induction of dog sperm capacitation by oviductal fluid. J. Vet. Med. Sci. 60: $197-202$

16. Kawakami, E., Naitoh, H., Ogasawara, M., Tamura, M., Hasegawa, J., Tsutsui, T. and Ogasa, A. 1991. Hyperactivation and acrosome reaction in vitro in spermatozoa ejaculated by cryptorchid dogs after orchiopexy. J. Vet. Med. Sci. 53: 447450.

17. Lee, C.N. and Ax, R.L. 1984. Concentrations and composition of glycosaminoglycans in the female bovine reproductive tract. J. Dairy Sci. 67: 2006-2009.

18. Lee, S.M., Clayton, M.K., Bushmeyer, S.M., First, N.L. and Ax, R.L. 1986. Glycosaminoglycans in ewe reproductive tracts and their influence on acrosome reactions in bovine spermatozoa in vitro. J. Anim. Sci. 63: 861-867.

19. Lenz, R.W. and Martin, J.L. 1988. Predicting fertility of dairy bulls by inducing acrosome reactions in sperm with chondroitin sulfates. J. Dairy Sci. 71: 1073-1077.

20. Mahi, C.A. and Yanagimachi, R. 1978. Capacitation, acrosome reaction, and egg penetration by canine spermatozoa in a simple defined medium. Gamete Res. 1: 101-109.

21. Mahmoud, A.I. and Parrish, J.J. 1996. Oviductal fluid and heparin induce similar surface changes in bovine sperm during capacitation: a flow cytometric study using lectins. Mol. Reprod. Dev. 43: 554-560.

22. Meizel, S. and Turner, K.O. 1986. Glycosaminoglycans stimulate the acrosome reaction of previously capacitated hamster sperm. J. Exp. Zool. 237: 137-139.

23. McCauley, T.C., Bellin, M.E. and Ax, R.L. 1996. Localization of a heparin-binding protein to distinct regions of bovine sperm. J. Anim. Sci. 74: 429-438.

24. McNutt, T., Rogowski, L., Vasilatos-Younken, R. and Killian, G. 1992. Adsorption of oviductal fluid proteins by the bovine sperm membrane during in vitro capacitation. Mol. Reprod. Dev. 33: 313-323.

25. Miller, D.J. and Hunter, A.G. 1986. Effect of osmolality and glycosaminoglycans on motility, capacitation, acrosome reaction, and in vitro fertilizability of bovine ejaculated sperm. J. Dairy Sci. 69: 2915-2924.

26. Morita, M., Miyamoto, H., Sugimoto, M., Sugimoto, N. and
Manabe, N. 1997. Alterations in cell proliferation and morphology of ampullar epithelium of the mouse oviduct during the estrous cycle. J. Reprod. Dev. 43: 235-241.

27. Murray, M.K. 1992. Biosynthesis and immunocytochemical localization of an estrogen-dependent glycoprotein and associated morphological alterations in the sheep ampulla oviduct. Biol. Reprod. 47: 889-902.

28. Parrish, J.J., Susko-Parrish, J.L., Winer, M.A. and First, N.L. 1988. Capacitation of bovine sperm by heparin. Biol. Reprod. 38: $1171-1180$.

29. Parrish, J.J., Susko-Parrish, J.L., Handrow, R.R., Sims, M.M and First, N.L. 1989. Capacitation of bovine spermatozoa by oviductal fluid. Biol. Reprod. 40: 1020-1025.

30. Parrish, J.J., Susko-Parrish, J.L., Uguz, C. and First, N.L. 1994. Differences in the role of cyclic adenosine 3,5-monophosphate during capacitation of bovine sperm by heparin or oviduct fluid. Biol. Reprod. 51: 1099-1108.

31. Ranganathan, S., Ganguly, A.K. and Datta, K. 1994. Evidence for presence of hyaluronan binding protein on spermatozoa and its possible involvement in sperm function. Mol. Reprod. Dev. 38: 69-76.

32. Rodriguez-Martinez, H., Larsson, B., Pertoft, H. and Kjellen, L. 1998. GAGs and spermatozoon competence in vivo and in vitro. In: Gametes: Development and Function (Lauria, A., Gandolfi, F., Enne, G. and Gianaroli, L. eds.), Serono Symposia, Roma.

33. Shamsuddin, M., Rodriguez-Martinez, H. and Larsson, B. 1993. Fertilizing capacity of bovine spermatozoa selected after swim-up in hyaluronic acid-containing medium. Reprod. Fertil. Dev. 5: 307-315.

34. Slotte, H., Akerlof, E. and Pousette, A. 1993. Separation of human spermatozoa with hyaluronic acid induces, and Percoll inhibits, the acrosome reaction. Int. J. Androl. 16: 349-354.

35. Suarez, S.S., Dai, X.-B., DeMott, R.P., Redfern, K. and Mirand, M.A. 1992. Movement characteristics of boar sperm obtained from the oviduct or hyperactivated in vitro. J. Androl. 13: $75-80$.

36. Talbot, T.L. and Chacon, R.S. 1981. A triple-stain technique for evaluating normal acrosome reactions of human sperm. $J$. Exp. Zool. 215: 201-208.

37. VandeVoort, C.A., Cherr, G.N. and Overstreet, J.W. 1997. Hyaluronic acid enhances the zona pellucida-induced acrosome reaction of macaque sperm. J. Androl. 18: 1-5.

38. Whiteman, P. 1973. The quantitative measurement of alcian blue-glycosaminoglycan complexes. Biochem. J. 131: 343350 . 